\title{
The Differentiation of Intestinal-Failure- Associated Liver Disease from Nonalcoholic Fatty Liver and Nonalcoholic Steatohepatitis
}

\author{
Alan L. Buchman, MD, MSPH${ }^{1}$ Bita V. Naini, MD² \\ ${ }^{1}$ Department of Surgery, University of Illinois, Chicago, Illinois \\ ${ }^{2}$ Department of Pathology, UCLA Medical Center, Los Angeles, \\ California \\ ${ }^{3}$ Bert Spilker and Associates LLC, Bethesda, Maryland
}

Bert Spilker, PhD, MD ${ }^{3}$

\author{
Address for correspondence Alan L. Buchman, MD, MSPH, \\ Department of Surgery, University of Illinois, Chicago, IL 60607 \\ (e-mail: a.buchman@hotmail.com).
}

Semin Liver Dis 2017;37:33-44.
Abstract
Keywords
- intestinal-failure- associated liver disease
- nonalcoholic fatty liver disease
- nonalcoholic steatohepatitis
- choline
- parenteral nutrition

Intestinal failure-associated liver disease (IFALD), formerly known as parenteral nutrition-associated liver disease has often been listed in textbooks as an example of nonalcoholic fatty liver disease (NAFLD). However, the etiology, pathophysiology, epidemiology, histology, and progression differ substantially between the conditions defined as NAFLD and the disease, IFALD. Therefore, IFALD should not be defined or considered as a type or a cause of nonalcoholic fatty liver or nonalcoholic steatohepatitis, but rather as a distinct disease.
Liver disease is prevalent in a high proportion of patients with intestinal failure receiving long-term parenteral nutrition (PN); the presentation of liver disease in patients receiving $\mathrm{PN}$ is different from that of nonalcoholic fatty liver disease (NAFLD). Intestinal-failure-associated liver disease (IFALD) has been listed as one presentation of NAFLD. ${ }^{1}$ However, here we will demonstrate substantial differences between IFALD and NAFLD in pathogenesis, risk factors, physiology, histology, epidemiology, and natural history. Although IFALD is similar to NAFLD in some respects, such as the presence of steatosis, the overall clinical presentation of IFALD is quite distinct from NAFLD. For example, IFALD is often characterized by low-plasma-free choline concentration, indicative of choline deficiency, hepatic steatosis with cholestasis, and rapid progression of the liver disease to hepatic failure, followed either by an intestine-liver transplant or death. In this review, nonalcoholic fatty liver and nonalcoholic steatohepatitis, abbreviated as NAFL/NASH, refer to both NAFL and $\mathrm{NASH}$, which together form the population of NAFLD.

Some experts believe that intestinal failure is an etiology that leads to NAFLD, and that intestinal failure is indistin- guishable from the more generic NAFLD associated with obesity, metabolic syndrome, and diabetes. On the contrary, a careful literature review reveals this dogma is incorrect and that IFALD is a specific disease with a different pathophysiology that is distinct from that of NAFLD. Moreover, IFALD is characterized by a characteristic histology that can often be differentiated from NAFLD in that IFALD consists of both steatosis and cholestasis (which could be referred to as steatocholestasis), whereas cholestasis is rarely noted in NAFLD. Both diseases have only a single common clinical characteristic, which is hepatic steatosis. IFALD and NAFLD occur and develop via different pathological mechanisms and in different patient populations. The vast majority of patients with NAFLD never progress to develop NASH and more severe liver disease, ${ }^{2}$ whereas in IFALD the progression to clinically significant liver disease is often rapid, and occurs in a substantial percentage of the adults and children on longterm PN, as discussed below.

Here we will distinguish IFALD as an objectively distinct form of liver disease, and present evidence that IFALD is highly associated with, and may possibly be explained by
Copyright $\odot 2017$ by Thieme Medical Publishers, Inc., 333 Seventh Avenue, New York, NY 10001, USA. Tel: +1(212) 584-4662. ISSN 0272-8087. 
choline deficiency, whereas NAFL/NASH is highly likely to be independent of choline deficiency. The frequency of choline deficiency in chronic PN patients is 80 to $85 \%$, 3,4 whereas choline deficiency has never been reported to our knowledge in either NAFL or NASH.

\section{Definitions}

\section{Intestinal Failure}

The term "intestinal failure" relates to a condition where a patient is unable to absorb sufficient energy, nutrients, and fluid to sustain life without receiving at least some PN. It was most recently defined in the literature as "the reduction of gut function below the minimum necessary for the absorption of macronutrients and/or water and electrolytes, such that intravenous supplementation is required to maintain health and/or growth." ${ }^{5}$ This situation occurs in a variety of malabsorption disorders such as short bowel syndrome, radiation enteritis, refractory sprue, and various motility disorders. Patients with intestinal failure who receive most of their caloric intake parenterally are defined in this article as PN patients. Long-term PN of a month or more often leads to liver disease, which may be severe, and is the most common complication of chronic PN associated with death in adults. ${ }^{6-9}$

\section{Intestinal Failure Associated Liver Disease}

Intestinal failure-associated liver disease has been defined in terms of hepatic enzyme abnormalities by the International Small Bowel Transplant Association (ISBTA), now known as the Intestinal Rehabilitation and Transplant Association (IRTA), which is a subdivision of the Transplantation Society. Their definition is "a persistent elevation of liver enzymes, alkaline phosphatase and $\gamma$-glutamyl transferase $\times 1.5$ above the upper limit reference range which persist for more than or equal to 6 months in adults and more than or equal to 6 weeks in children." 10 Therefore, cholestasis, as defined by the elevation of these liver enzymes (as well as by the histology discussed below), is a very common finding in the majority of patients with IFALD. In terms of biomarkers, imaging, and pathology, early IFALD is defined by the IRTA as "total bilirubin will be less than $3 \mathrm{~g} / \mathrm{L}$, and hepatic ultrasound will show an echogenic appearance. If a liver biopsy is performed, up to $25 \%$ of liver parenchyma will demonstrate steatosis, or up to $50 \%$ portal tracts will show fibrotic changes."10 Microvesicular steatosis is often noted in biopsy samples from patients with IFALD and is also a distinguishing characteristic. These reasonably accepted surrogate markers (i.e., cholestasis and steatosis as described above) for IFALD have been well accepted within the community of professionals who treat patients with this disorder. It is important to note that IFALD was formerly termed parenteral-nutrition-associated liver disease until 2002, when it was recognized that choline may be an important factor in the etiology of IFALD in PN patients, as opposed to the more complex theories that claimed IFALD was related to parenteral toxicities in a parenteral admixture. ${ }^{11-13}$

\section{Nonalcoholic Fatty Liver Disease}

Nonalcoholic fatty liver disease is defined by the guidelines of the American Gastroenterological Association (AGA), the
American Association for the Study of Liver Disease (AASLD), and the American College of Gastroenterology (ACG), to include those patients who have simple steatosis (NAFL), as well as those patients who have steatosis plus hepatitis (i.e., NASH); both are described below. ${ }^{14}$ The presence of hepatic steatosis as outlined in the guidelines is based on either imaging or histology. Secondary causes of fat accumulation in the liver such as significant ethanol consumption, use of known steatogenic medications, PN, or hereditary disorders must be excluded to make the diagnosis for NAFLD. NAFLD consists of the summation of those patients with NAFL and those with NASH (NAFL/NASH). ${ }^{14}$

\section{Nonalcoholic Fatty Liver}

As described by a multisociety consortium (AGA, AASLD, ACG), the presence of NAFL indicates the absence of recent or ongoing consumption of significant amounts of ethanol, and requires the exclusion of $\mathrm{PN}$, various steatogenic medications, and various hereditary disorders. ${ }^{14}$ It is defined in terms of histological abnormalities in a report that summarized conclusions from an AASLD workshop held in 2009 to define and develop endpoints for clinical trials in NAFL/NASH. Specifically, NAFL was defined as "> $>$ \% macrovesicular steatosis as evaluated by light microscopic examination of a hematoxylin and eosin stained liver section (4-5 $\mu \mathrm{m}$ thick) under a $10 \times$ objective lens." ${ }^{15,16}$ In addition, there is no evidence of hepatocellular injury as defined by hepatocyte ballooning, ${ }^{14}$ and there is no evidence of inflammation. Finally, cholestasis is rarely, if ever, observed in patients with NAFL.

\section{Nonalcoholic Steatohepatitis}

The presence of NASH indicates the absence of recent or ongoing consumption of significant amounts of ethanol, and requires the exclusion of PN, and various hereditary disorders. ${ }^{14}$ It is defined histologically as "the presence of $>5 \%$ macrovesicular steatosis, inflammation and liver cell ballooning, typically with a predominately centrilobular (acinar zone 3) distribution in adults."15 This indicates the presence of steatohepatitis. Cholestasis is rarely, if ever, observed in patients with NASH.

Note that patients with NASH are not receiving PN unless there is an unrelated disease for which PN is indicated and used.

\section{Cholestasis}

Cholestasis is defined as a decrease in bile flow due to impaired secretion of bile by hepatocytes or due to obstruction of bile flow in intra- or extrahepatic bile ducts. (http://emedicine. medscape.com/article/927624-overview; accessed July 31, 2015). It may be diagnosed histologically with a liver biopsy supported by elevations of serum alkaline phosphatase and aspartate aminotransferase or alanine aminotransferase. Cholestasis is observed in a majority of patients with IFALD and rarely, if ever, observed in NAFL/NASH patients. $6,7,9,11,17-23$

\section{Histology}

\section{Intestinal Failure-Associated Liver Disease}

The presence of cholestasis is a hallmark of IFALD $6,7,9,17,24$ in both pediatric and adult patients, and is not associated with 
NAFLD in the absence of another underlying condition. Bowyer et al found that five of nine long-term PN patients in whom liver biopsies were performed had cholestasis histologically and four of the five cholestatic patients had concomitant hepatic steatosis. ${ }^{6}$ Stanko et al biopsied four long-term PN patients who had elevated serum alkaline phosphatase and hepatic aminotransferases and found histological cholestasis and concomitant steatosis in all four patients. ${ }^{7}$ Among a group of 90 patients who required longterm PN, Cavicchi et al observed that $72 \%$ had developed biochemical evidence of cholestasis (defined as $1.5 \times$ the normal upper value for at least two of three of the following biochemical liver tests: serum $\gamma$-glutamyltransferase, alkaline phosphatase, and conjugated bilirubin that persisted for at least 6 months) after a median of 6 years of PN (range 6-198 months). ${ }^{9}$ Sixty-three (70\%) of the 90 patients had at least one liver biopsy. Seventy-six percent of these patients had histological evidence of cholestasis; histological cholestasis was found in an additional three patients who had no biochemical evidence of cholestasis.

A hallmark of IFALD is the concomitant presence of cholestasis and steatosis (-Fig. 1), although cholestasis may be evident only biochemically. In the Cavicchi et al study, hepatic steatosis (macro) was evident in $63 \%$ of patients with chronic (defined as at least 6 months in duration) biochemical cholestasis, as well as in all patients who did not have chronic biochemical cholestasis. ${ }^{9}$ Forty (63\%) of the patients who underwent liver biopsy also had microsteatosis or phospholipidosis. It cannot be ascertained from the data whether any of these represented unique patients from those previously reported from the same study with macrosteatosis. In a retrospective review of 89 patients (including 53 infants) who received long-term PN and had liver biopsies, Naini and Lassman found $67 \%$ of patients had histological evidence of cholestasis and $58 \%$ had steatosis, although this finding was not differentiated into those with just macro- or microvesicular steatosis or both. ${ }^{17}$ In the older children and adults (with

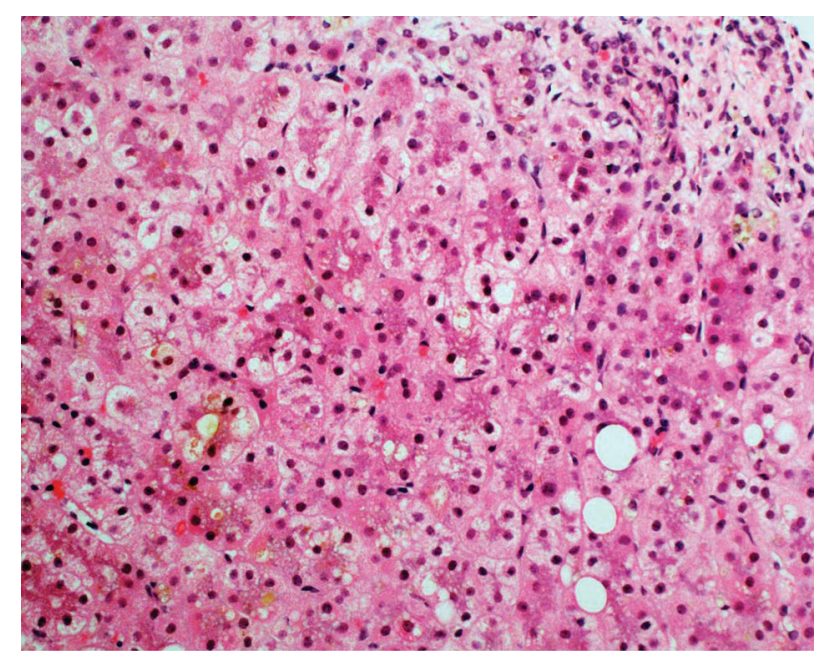

Fig. 1 Intestinal failure-associated liver disease (IFALD) with mixed cholestasis as well as steatosis in zone 1.The portal tract on the top right corner shows features of biliary obstruction with portal edema and ductular reaction, a hallmark of IFALD. the 36 infants excluded), $74 \%$ of the patients who had a liver biopsy had elevated serum alkaline phosphatase and $81 \%$ had elevated hepatic aminotransferase concentrations.

Patients with IFALD may exhibit an uncommon and often characteristic subtype of steatosis, namely a combination of both macro- and microvesicular, ${ }^{9}$ which is not generally observed in NAFL/NASH in the absence of chemical exposure or other toxins, and a low rate of steatohepatitis. It is notable that microvesicular steatosis in hepatic cells is observed in a majority of IFALD patients. However, microvesicular steatosis is not a common histological finding of NAFL associated with obesity, diabetes mellitus, metabolic syndrome, etc. ${ }^{15,25} \mathrm{Mi}-$ crovesicular fatty degeneration refers to the presence of small intracytoplasmic fat vacuoles (liposomes) that accumulate in the hepatocyte. Many of these small fat vacuoles are diffusely dispersed throughout the hepatocyte cytoplasm, and the nucleus remains centrally placed.

Macrovesicular fatty degeneration occurs when there is a single, bulky fat vacuole that distends the hepatocyte and pushes the nucleus and cytoplasm to the side. This condition is termed "macrovesicular steatosis." Macrovesicular steatosis is commonly associated with NAFL. ${ }^{15,25}$ From a pathological perspective, macrovesicular steatosis implies a stable equilibrated excess of hepatic triglycerides that in the majority of patients, does not have a negative impact on cellular function that could lead to disease. On the other hand, microvesicular steatosis implies ongoing, active lipid turnover and metabolic instability, and mitochondrial injury. ${ }^{26-30}$ Microvesicular fatty change is therefore a more ominous histological finding than is its large-droplet counterpart found in both NAFL and NASH (http://tpis.upmc.com/tpislibrary/dlp/Chap7frame.html; accessed July 30, 2015).

\section{Histopathology}

As mentioned, cholestasis is the most common histopathological finding in IFALD regardless of the patient's age. ${ }^{6,7,9,11,17-23}$ Histological findings ( - Table 1 ) include

Table 1 Comparison of histological characteristics of IFALD vs. NAFLD

\begin{tabular}{|l|l|}
\hline IFALD & NAFLD \\
\hline Cholestasis & No cholestasis \\
\hline Macro- and microsteatosis & $\begin{array}{l}\text { Predominately } \\
\text { macrosteatosis }\end{array}$ \\
\hline Steatosis in zone 1 & Steatosis in zone 3 \\
\hline $\begin{array}{l}\text { Features of biliary obstruction } \\
\text { (portal inflammation, edema, } \\
\text { ductular proliferation); } \\
\text { ductopenia }\end{array}$ & $\begin{array}{l}\text { No features of biliary } \\
\text { obstruction or ductopenia }\end{array}$ \\
\hline Steatohepatitis rare & Steatohepatitis common \\
\hline “Jigsaw” pattern of fibrosis & $\begin{array}{l}\text { Sinusoidal fibrosis; } \\
\text { ballooned hepatocytes } \\
\text { with Mallory-Denk bodies }\end{array}$ \\
\hline
\end{tabular}

Abbreviations: IFALD, intestinal failure-associated liver disease; NAFLD, nonalcoholic fatty liver disease. 


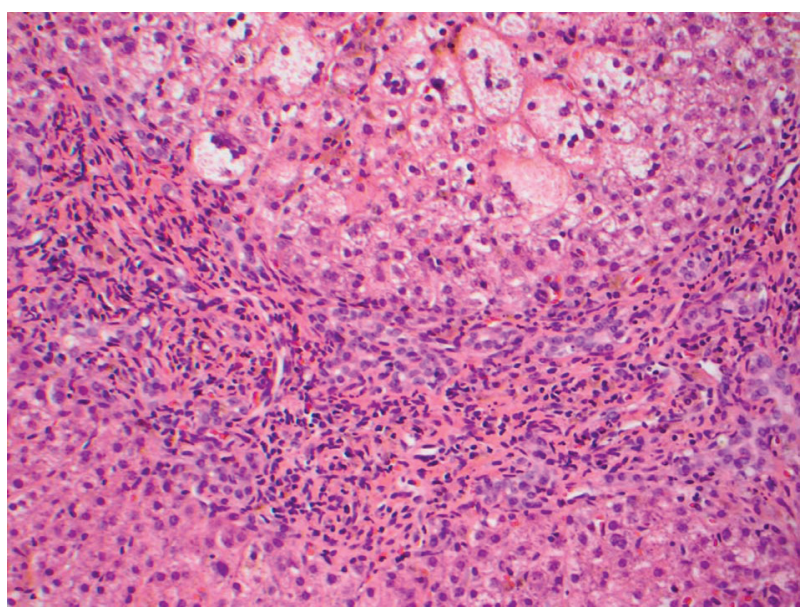

Fig. 2 Intestinal failure-associated liver disease showing features of biliary obstruction (i.e., portal expansion by neutrophils, portal edema, and ductular reaction.

- Features of biliary obstruction that are often present $(65 \%$ of patients). ${ }^{17}$ The underlying reason behind this histology is the presence of biliary sludge that occurs in nearly all patients who undergo PN treatment. These include (1) mixed portal inflammation with prominence of neutrophils, (2) portal edema, and (3) ductular proliferation (-Fig. 2). Ductopenia occurs in $~ 10 \%$ of patients ${ }^{17}$ it refers to the absence of bile ducts in more than $50 \%$ of the portal tracts.

- Steatosis is more common in older children and adults than in infants and neonates. The steatosis is macrovesicular and is distributed in zone 1 (periportal), at least initially, ${ }^{20,23}$ although many of the patients also have a microvesicular form. In contrast, steatosis and steatohepatitis in NASH are more commonly found in zone 3 (pericentral) (-Fig. 3). Extremely few reports have described the occurrence of microvesicular steatosis in NASH, ${ }^{25,31,32}$ although Cotrim et al included primarily subjects with hepatotoxic chemical exposure, which is not a common etiology of NASH. ${ }^{31}$ Tandra et al found evidence of microsteatosis in $10 \%$ of 1,022 liver biopsies

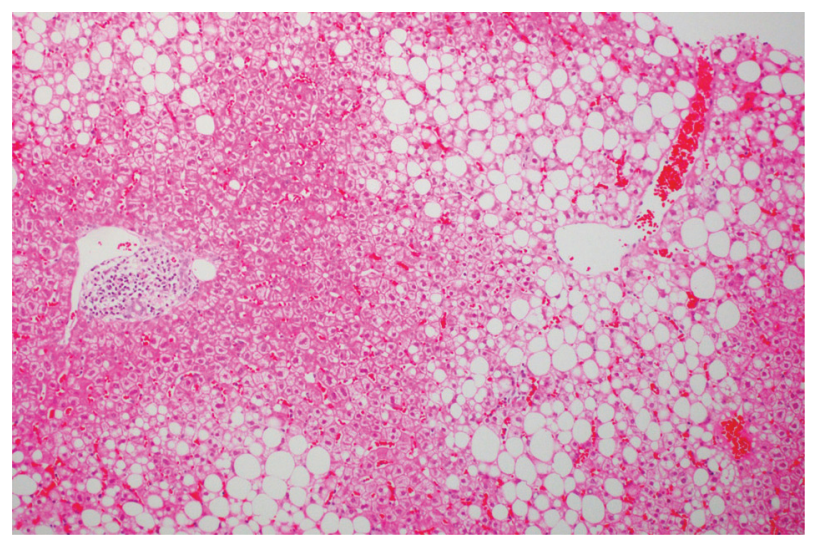

Fig. 3 Nonalcoholic fatty liver disease showing macrovesicular steatosis in zone 3. from adults that participated in two studies conducted by the NASH Clinical Research Network. ${ }^{25}$

- Rarely, steatohepatitis has been reported in IFALD adults, particularly in early reports of IFALD ${ }^{6}$; isolated steatohepatitis without cholestasis is rarely observed in these patients. ${ }^{9,17}$ If steatohepatitis does occur in an IFALD patient, it is usually accompanied by other characteristics of IFALD, such as cholestasis. In contrast, the presence of steatohepatitis, without cholestasis, is the primary finding that defines NASH.

- Fibrosis leading to cirrhosis occurs in more advanced cases of IFALD and begins with portal expansion.

- Ballooned hepatocytes with Mallory-Denk bodies, lobular inflammation with lymphocytes and occasional spotty areas of necrosis, and sinusoidal fibrosis around ballooned hepatocytes are characteristically encountered in NAFL/ NASH ( - Fig. 4). ${ }^{15,33-38}$

- A biliary pattern of fibrosis/cirrhosis (with its characteristic geographic "jigsaw" pattern of fibrosis) is a much more common pattern of end-stage liver disease secondary to IFALD than is regular cirrhosis secondary to NASH or chronic hepatitis (-Fig. 5). In IFALD, fibrosis begins via
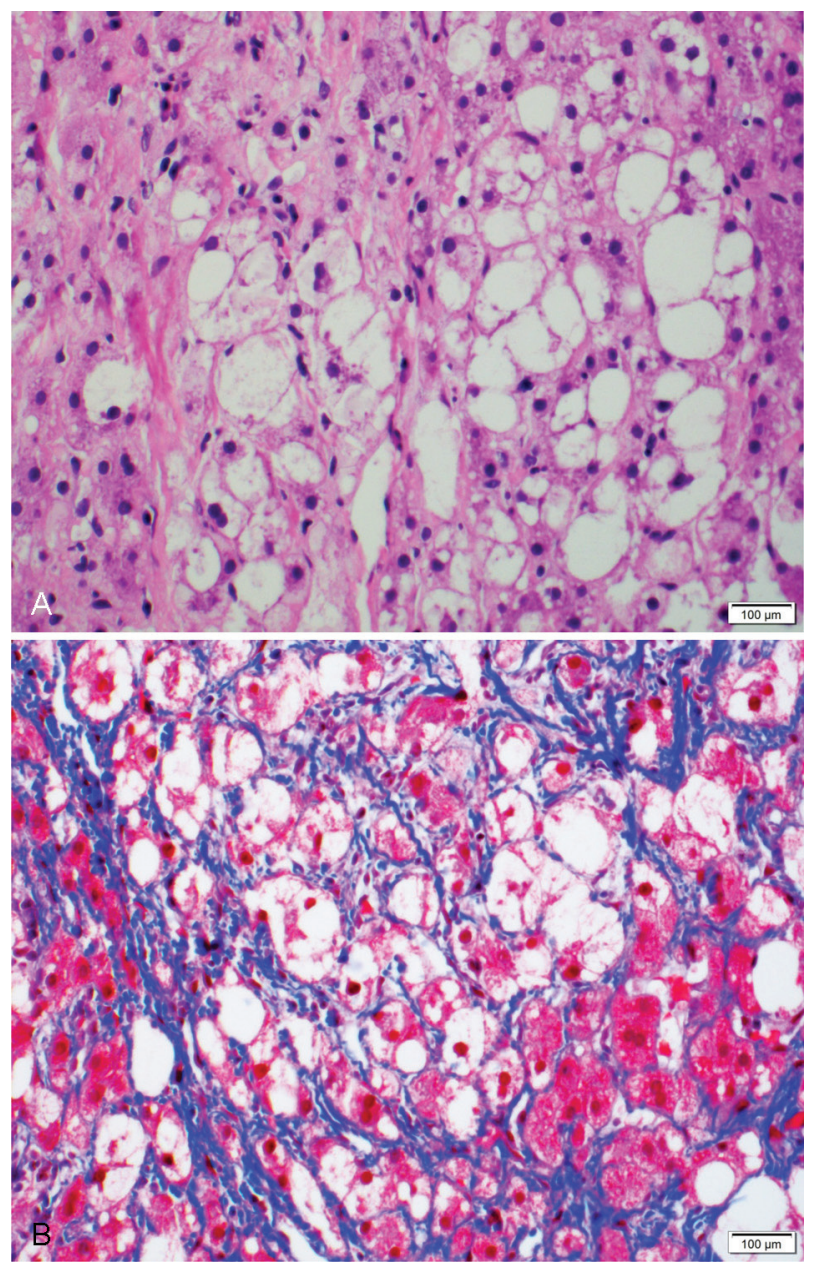

Fig. 4 (A) Nonalcoholic steatohepatitis (NASH) showing steatosis as well as ballooned hepatocytes and Mallory-Denk bodies. (B) NASH showing pericellular/sinusoidal fibrosis around ballooned hepatocytes. 


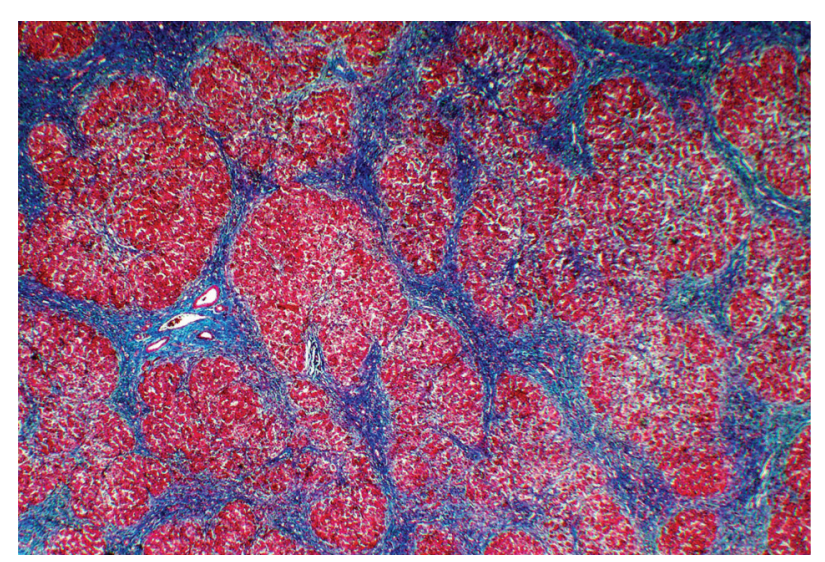

Fig. 5 Intestinal failure-associated liver disease with biliary pattern of cirrhosis.

portal expansion and progresses to periportal fibrosis and extends later to portal-portal bridging fibrosis whereas fibrosis in NASH occurs predominately in zone 3.

\section{Pathophysiology of Intestinal Failure-Associated Liver Disease}

It was reported in 1972 that the normal hepatic transsulfuration pathway for the metabolism of orally administered methionine into choline and other products (e.g., cysteine) did not function at all when the methionine was provided intravenously, such as via PN. ${ }^{39}$ In this study, the plasma cystine concentration was substantially decreased in normal volunteers when methionine was provided intravenously when compared with the cysteine concentrations following enteral methionine administration. Plasma cystine was measured in this study rather than plasma cysteine because the former is a much more stable compound. Cystine is formed from the oxidation of two cysteine molecules. The blockage of normal hepatic transsulfuration pathway when nutrients were given intravenously was also reported by Chawla et al in 1985 and Sentongo et al in $2010 .{ }^{40,41}$ Specifically, choline, as well as other products of this pathway was significantly reduced in the plasma of hospitalized children and adults who required intravenous feeding, and was lowest in those patients with cirrhosis, who have a substantially decreased ability to metabolize methionine and synthesize choline. The reasons for this blockage appear to be related to the fact that normal metabolism of methionine to choline and other products of the hepatic transsulfuration pathway requires first-pass metabolism, which occurs only when methionine is absorbed enterally and transported via the portal circulation. ${ }^{42}$ Therefore, unlike patients who can eat and absorb methionine-containing food, those who receive all of their nutrition from PN are unable to fully utilize the methionine contained in PN to synthesize choline. In fact, human studies have demonstrated that plasma methionine concentrations range from high normal to elevated in PN patients, illustrating the result of insufficient hepatic methionine metabolism. ${ }^{40,43,44}$
Plasma choline concentration is therefore reduced in IFALD patients because

1. They do not absorb sufficient choline from the diet due to their malabsorption.

2. They do not obtain choline from PN solutions, as none is supplemented outside of the miniscule amount found naturally in lipid emulsions.

3. They are unable to synthesize choline from the primary substrate, methionine, which is present in PN solutions. The reason for the failure of this pathway to function in IFALD patients is discussed above.

In patients with IFALD, their below-normal plasma-free choline concentration significantly correlates both with hepatic biochemical abnormalities as well as with liver tissue abnormalities in humans. ${ }^{3,45-48}$ Plasma-free choline was below normal in 33 of 41 long-term PN patients in one study ${ }^{3}$ and in 18 of 21 in another ${ }^{4}$ and the correlation between the plasma-free choline concentration and degree of hepatic steatosis in the Buchman study was $r=-0.81, p<0.001 .^{46}$

Several published studies show that animal models are consistent with these observations in humans. For example, in the short-term dietary manipulation studies of rodents in which choline was artificially repleted in choline-deficient animals, it is likely that these were really models of IFALD. This is because repletion relates primarily to a state of choline deficiency as found in IFALD, and not to one of choline excess as is often found in NAFL. ${ }^{49}$ This further suggests that the methionine-choline-deficient rodent model used to simulate NASH according to some authors ${ }^{50,51}$ is in actuality a model of IFALD and not a model simulating NASH. Similarly, artificially induced IFALD will result from choline deficiency, for example, when rodents are maintained on choline-free PN as their sole source of nutrition. ${ }^{52}$ It is of note that this choline-free PN model shows that a method of inducing liver disease is to cause choline deficiency; however, in the short-term choline-deficient diet model, repletion of choline was utilized as a treatment regime. Both of these models provide evidence that insufficient choline has a causal link to the development of hepatic steatosis, and choline repletion can reverse the impact of a choline-deficient diet. Furthermore, mice provided with choline-deficient diets do not develop the insulin resistance associated with $\mathrm{NAFL} / \mathrm{NASH},{ }^{53,54}$ although one study suggested insulin resistance did develop in rats fed a cholinedeficient diet. ${ }^{55}$ However, those rats also became obese, which is certainly not characteristic of the long-term PN patient.

Recently, a true model of NASH was created by feeding LCT $\alpha(-/-)$ mice a choline-sufficient, but high-fat diet. ${ }^{56}$ Impaired hepatic phosphatidylcholine synthesis occurred in these mice due to the liver-specific elimination of phosphocholine cytidylyltransferase. The elimination of phosphocholine cytidylyltransferase prevented the metabolism of choline in this pathway and resulted in decreased hepatic very-lowdensity lipoprotein secretion, which in turn resulted in the development of hepatic steatosis, although without immediately causing steatohepatitis. However, within a week of feeding mice this high-fat diet, NASH did develop. Treatment with parenteral choline was attempted, but this failed to 
either prevent or to treat the steatohepatitis. This is further evidence that choline deficiency does not cause NASH; therefore, choline therapy also does not appear to be a suitable treatment in patients with NASH. These animal studies provide further evidence of the differences between IFALD and NASH.

In contrast to IFALD, where the plasma-free choline concentration is very low, suggestive of choline depletion, the plasma-free choline concentration is normal to elevated in NAFL/NASH. ${ }^{57-59}$ Moreover, the concentration of choline increases rather than decreases as the severity of hepatic histology worsens $(n=65) .{ }^{57}$ In fact, Nehra et al $^{59}$ concluded, "Neither choline deficiency nor celiac sprue by anti-endomysial antibody testing was associated with NASH" in the Mayo Clinic NASH population. Furthermore, Imajo et al found plasma-free choline concentrations were higher in patients with NASH $(n=110)$ than in other liver diseases. ${ }^{57}$ Therefore, there is no evidence that the hepatic transsulfuration pathway (which is intimately involved with choline synthesis) is impaired in patients with NASH.

Therefore, in contrast to the three reasons for observing choline deficiency in IFALD patients (see above), NAFL/NASH patients have a normal or elevated choline level because they

1. Do not have malabsorption of nutrients including choline

2. Are not receiving PN and are able to maintain an enteral diet with sufficient choline

3. Have a normal hepatic transsulfuration pathway and are able to synthesize choline from an orally consumed methionine substrate, whereas IFALD patients have malabsorption and cannot absorb and utilize methionine from their diet

Therefore, the evidence to support the theory that choline deficiency contributes to the etiology of IFALD includes

1. The use of a choline-deficient diet in rodents to create a liver disease model

2. Choline deficiency results in reduced synthesis of phospholipids, lipid transport, and increased intracellular lipids, and the development of hepatic steatosis in animal models.

3. The very high frequency of choline deficiency in PN patients
4. The reduction of steatosis and other markers of liver disease in choline-supplemented PN patients and recurrence of steatosis when choline is removed from their PN in preliminary studies

Although in rodent models, choline deficiency is associated with mitochondrial abnormalities, ${ }^{29,60}$ the fact that choline levels in patients with NASH are normal or even elevated precludes choline deficiency as a cause for the mitochondrial abnormalities encountered in NASH. ${ }^{61}$

\section{Risk Factors and Epidemiology}

\section{Risk Factors and Etiologies/Comorbidities}

A diagnosis of IFALD requires that the patient is receiving PN, whereas a diagnosis of NAFL/NASH prohibits PN. The following conditions/risk factors are generally associated with the presence of NAFL or NASH, but not IFALD or in patients who require $\mathrm{PN}$ (-Table $\mathbf{2}$ ):

- Obesity (body mass index [BMI] > 30): The prevalence of hepatic steatosis in very obese patients (BMI $>40$ or $>35$ with comorbidities) may be as high as $85 \%,{ }^{62-65}$ whereas the average BMI of PN patients is stable at an approximate mean value of $21 .^{66}$ This indicates that obesity rarely, if ever, occurs during PN. Recent data have shown that although NASH may occur in a nonobese population, it is uncommon, and when it occurs, it is still associated with insulin resistance and dyslipidemia, in addition to increased waist circumference. In addition, nonobese patients with NAFL rarely if ever progress to NASH or advanced fibrosis. ${ }^{67}$ Therefore, the nonobese patient with NAFL remains distinct from a patient with IFALD.

- Type II diabetes mellitus with insulin resistance/hyperinsulinemia: Upwards of $75 \%$ of those with type 2 diabetes may have hepatic steatosis, ${ }^{68-71}$ whereas there are no reports that the frequency of type II diabetes in IFALD is different than that in the general population. Studies in humans have reported that insulin resistance is actually reduced in hospitalized patients who required $\mathrm{PN}^{22,73}$ One study reported that although PN use was not associated with decreased insulin resistance, PN had no effect. ${ }^{74}$

Table 2 Comparison of pathophysiology and risk factors of IFALD vs. NAFLD

\begin{tabular}{|l|l|}
\hline IFALD & NAFLD \\
\hline Severe nutrient and medication malabsorption & No nutrient or medication malabsorption \\
\hline Parenteral nutrition required & Patients on parenteral nutrition excluded \\
\hline$\downarrow$ Plasma-free choline concentration & Normal or $\uparrow$ plasma-free choline concentration \\
\hline No obesity; BMI normal to low & Obesity prevalent \\
\hline$\downarrow$ Plasma cholesterol concentration & Hyperlipidemia \\
\hline Metabolic syndrome not reported & Metabolic syndrome common \\
\hline No insulin resistance & Insulin resistance present \\
\hline No known cause by medications & Several medications can cause NAFLD \\
\hline
\end{tabular}

Abbreviations: BMI, body mass index; IFALD, intestinal failure-associated liver disease; NAFLD, nonalcoholic fatty liver disease. 
Furthermore, patients who have cycled PN (i.e., periods on and off PN during a 24-hour period) have an appropriate insulin response to sustained hyperglycemia, and insulin sensitivity is not affected. ${ }^{75}$

- Hyperlipidemia: Approximately a third of patients with hyperlipidemia have NAFL. ${ }^{76}$ One well-characterized case series reported a prevalence of hyperlipidemia (increased triglycerides, total cholesterol, or high-density lipoprotein) in $96 \%$ of patients with NASH. ${ }^{77}$

- Metabolic syndrome ${ }^{78-80}$ : Metabolic syndrome has not been reported in long-term PN patients, but is common in NAFLD.

- Drugs: NAFL/NASH has been associated with methotrexate, amiodarone, tetracycline, valproic acid, tamoxifen, and nucleoside analogues, as well as some toxins such as carbon tetrachloride, perchloroethylene, ethyl bromide, and petrochemicals. ${ }^{1}$

These common etiologies of NAFL/NASH (e.g., obesity, metabolic syndrome, hyperlipidemia, and noninsulin-dependent diabetes mellitus [NIDDM]) are rarely reported or observed in cases of long-term PN. For example, obesity and metabolic syndrome have not been reported in longterm PN patients, although short bowel syndrome and a subsequent requirement for long-term $\mathrm{PN}$ is a rare but recognized complication of bariatric surgery. ${ }^{81}$ Blood cholesterol concentrations are generally lower than normal, even strikingly so (e.g., $<100 \mathrm{mg} / \mathrm{dL}$ ) in patients who receive longterm PN. ${ }^{82,83}$ However, blood cholesterol is usually substantially elevated in patients with NASH. It is also unusual to encounter patients with NIDDM on long-term PN (no such patients were reported in the medical literature). In addition, NAFL/NASH patients often have normal or elevated plasmafree choline concentration. ${ }^{57,58}$ These characteristics of NAFL/ NASH further serve to distinguish IFALD from NAFL/NASH.

\section{Prevalence}

Although the precise number of patients who are receiving long-term PN in the United States is unknown, it has been estimated that this number is approximately 50,000 individuals. Data derived from the OASIS Registry sponsored by the Oley Foundation (Albany, NY) registry and Medicare home PN (HPN) payments from 1989 to 1992 (the most recent data available) were used to estimate the number of Medicare HPN consumers at a rate of $\sim 238$ per million. ${ }^{84}$ Because Medicare recipients comprised $25 \%$ of the population sample, it was further extrapolated that in 1992 there were 40,000 patients who received HPN in the United States. ${ }^{84}$ However, it was also noted that this number included many cancer patients who received short-term PN. ${ }^{84,85}$ In fact, the annual growth in HPN of $8 \%$ was primarily due to the increased number of cancer patients being discharged from the hospital on HPN. The number of new patients with long-term disorders such as short bowel syndrome was relatively constant. Therefore, based on the above indication of prevalence of IFALD among PN patients and the Cavicchi et al data of IFALD prevalence $(60 \%$ after 2-4 years), the estimated total number of patients (including infants and children) with IFALD is as great as $50,000 .{ }^{9}$ It is also noted that children also make up a substantial number of long-term PN patients, although their exact percentage of the total HPN population is unknown. We suggest that the number of adults with IFALD is likely to be between 5,000 and 20,000. Therefore, IFALD is a rare disease according to the U.S. Food and Drug Administration's definition of a rare disease, which is based on having a prevalence of fewer than 200,000 patients in the United States.

\section{Natural History and Prognosis}

Intestinal-failure-associated liver disease affects a very limited number of patients in comparison to NAFL/NASH, but is more rapidly progressive and results in a substantially higher mortality rate ( - Table 3 ). Cavicchi et al reported that $65 \%$ of their 90 patients developed IFALD, manifested by chronic cholestasis after a median period of 6 months, and their disease progressed over time to more severe liver disease (-Table 4). ${ }^{9}$ Of these IFALD patients, $34 \%$ (22\% of all 90 in the study) died due to end-stage liver disease (ESLD), as a result of IFALD. A full $50 \%$ of their patients developed complicated liver disease, which was defined as portal hypertension, portal fibrosis or cirrhosis on biopsy, total serum bilirubin concentration $>3.5 \mathrm{mg} / \mathrm{dL}$ for at least one month, ascites, hepatic encephalopathy, variceal hemorrhage, or a factor $\mathrm{V}$ concentration $<50 \%$ after 6 years of PN.

Stanko and colleagues reported that four of six patients with short bowel syndrome during the time they received PN developed progressively increased hepatic aminotransferase and alkaline phosphatase concentrations, and all four had steatosis, cholestasis, and periportal inflammation. Further, all died within one year of diagnosis. ${ }^{7}$ Chan et al found $15 \%$ of their 42 adult patients on long-term PN at the Beth Israel Deaconess program in Boston developed IFALD that progressed to ESLD, and all died within a relatively short period. ${ }^{8}$ A survey of European intestinal failure centers found liver failure was the complication of PN that had the greatest risk of

Table 3 Comparison of epidemiology and natural history of IFALD vs. NAFLD

\begin{tabular}{|l|l|}
\hline IFALD & NAFLD \\
\hline $65 \%$ Prevalence in the long-term PN population & $<1 \%$ Prevalence in long-term PN patients \\
\hline $15-34 \%$ death rate within $1^{8}-4^{9}$ y & Rapid onset of death is extremely rare postdiagnosis \\
\hline Cirrhosis develops in as little as 3-5 mo after chronic PN initiated & Cirrhosis takes $10-20$ y to develop \\
\hline
\end{tabular}

Abbreviations: IFALD, intestinal failure-associated liver disease; NAFLD, nonalcoholic fatty liver disease; PN, parenteral nutrition. 
Table 4 Progression of IFALD in 90 Patients on Long-Term PN ${ }^{\mathrm{a}}$

\begin{tabular}{|l|l|l|}
\hline $\begin{array}{l}\text { \# y } \\
\text { on PN }\end{array}$ & $\begin{array}{l}\text { Prevalence of } \\
\text { IFALD (\%) }\end{array}$ & $\begin{array}{l}\text { Prevalence of } \\
\text { severe liver disease }^{\mathbf{b}} \text { (\%) }\end{array}$ \\
\hline 2 & 55 & 26 \\
\hline 4 & 64 & 39 \\
\hline 6 & 72 & 50 \\
\hline 8 & NA & 53 \\
\hline
\end{tabular}

Abbreviations: IFALD, intestinal failure-associated liver disease; NA, not applicable; NAFLD, nonalcoholic fatty liver disease; PN, parenteral nutrition.

aTotal number of deaths from IFALD at 8 years was $22 \%$.

${ }^{\mathrm{b}} \mathrm{A}$ diagnosis of severe liver disease was defined by (1) extensive portal fibrosis (grade 2), (2) cirrhosis (grade 3), (3) portal hypertension, (4) ascites, (5) variceal hemorrhage, (6) portal hypertensive bleeding, (7) hepatic encephalopathy, and/or (8) liver failure (factor $V \leq 50 \%$ ).

death-substantially greater than that from infection. ${ }^{86}$ In PN patients, cirrhosis has been reported to develop in neonates in as little as $3^{21}$ or 5 months, ${ }^{17}$ although progression is slower in adults. ${ }^{9}$ This compares with 10 to 20 years that it takes for patients with NASH to develop to cirrhosis. Intestinal-failureassociated liver disease therefore represents a more rapidly progressive and potentially fatal deterioration of hepatic function than is observed in either NAFL or NASH. End-stage liver disease, with or without cirrhosis, usually leads to liver transplantation, small bowel transplantation, or death. In IFALD, biliary cirrhosis (with its geographic "jigsaw" pattern of fibrosis) is a more common pattern of ESLD, than is cirrhosis secondary to NASH. This type of biliary cirrhosis is secondary to cholestatic injury. Animal studies have shown bile flow is decreased during PN therapy. ${ }^{87-89}$ Biliary sludge formed secondary to bile stasis is a well-described complication of PN. ${ }^{90,91}$ Messing et al reported bile sludge to be present in all adult patients who had been on PN for more than 6 weeks. ${ }^{90}$ Animal data suggest that the provision of choline increases bile flow. ${ }^{92}$

Impending liver failure is the single most common indication for isolated small intestinal transplantation. ${ }^{93}$ Liver failure during long-term PN requires a combined liver-intestine transplant, given that continued malabsorption of nutrients will lead to recurrent and steadily worsening hepatic insult. Isolated intestinal transplantation has led to the reversal of IFALD, substantiating the role of nutrient malabsorption as a (or the) primary cause for IFALD. ${ }^{12,94}$ On the other hand, there is no medical basis for considering intestinal transplantation as a method to treat NASH because there is no evidence of malabsorption in NASH. The malabsorption of essential nutrients is the primary reason why choline deficiency occurs in PN-dependent patients, but not in patients with NAFL or NASH.

In contrast, current medical opinion suggests NAFL can be considered as the hepatic manifestation of metabolic syndrome, and the simple steatosis that makes up NAFL does not affect survival. ${ }^{95}$ For example, Adams et al found that only $5 \%$ of 440 patients with NAFL developed cirrhosis after a mean follow-up time of 7.6 years ${ }^{96}$-a far lower progression rate over a much longer period than is the case with IFALD as noted above. In this study, only $1.7 \%$ of deaths in patients who went on to develop NASH were related to liver disease (compared with $22 \%$ in two studies of IFALD patients noted above). In an even longer study, only a single patient out of 109 patients with NAFL developed cirrhosis, and it took over 16 years. It was clear that the survival of patients in that group did not significantly differ from the normal population. ${ }^{97}$ In another study, only 6 of 92 (6.5\%) patients with NAFL developed cirrhosis during a mean follow-up of 13.4 years. ${ }^{98}$ Notwithstanding these observations, one recent study suggested that potentially up to $40 \%$ of patients with NAFL ( $N=108$ in this study) may progress to NASH after a mean of nearly 7 years, although progression to cirrhosis and death was not described. ${ }^{99}$ However, even the higher progression rate for NAFL in this one study does not indicate a prognosis or progression at the high rate that is evident in patients with IFALD.

Although most patients with NAFL have pure hepatic steatosis without inflammation and are reported to have a benign clinical course, 15 to $25 \%$ of those who progress from simple steatosis to NASH continue to progress to cirrhosis and its complications over a relatively long period of 10 to 20 years (http://www.clevelandclinicmeded.com/medicalpubs/diseasemanagement/hepatology/nonalcoholic-fatty-liver-disease/ Default.htm; accessed July 30, 2015). Further, once simple steatosis has progressed to $\mathrm{NASH}$, additional progression is much more protracted than that observed in patients with IFALD, and only a minority of patients with NASH eventually develop more severe liver disease. The literature also demonstrates that survival is substantially greater in patients with NAFLD, including those who progress to NASH, when compared with patients with IFALD.

\section{Diagnosis of IFALD Based on Clinical History, Hepatic Enzymes, Histology, and Plasma- Free Choline Concentration}

A patient is considered to be diagnosed with IFALD if he or she

1. Requires long-term PN, and

2. Has evidence of cholestasis in a liver biopsy or by elevated alkaline phosphatase liver isoenzyme, and/or

3. Has evidence of steatosis in a liver biopsy or by imaging techniques, and

4. Has a low plasma-free choline concentration, and

5. Has no major risk factor for NAFL/NASH (i.e., obesity, metabolic syndrome, hyperlipidemia, insulin resistance, NIDDM) (B.V. Naini, 2015, Personal Communication).

Alkaline phosphatase was found to be a validated and reasonably accepted biochemical indicator of cholestasis. ${ }^{100}$ Elevation of the alkaline phosphatase liver isoenzyme had a sensitivity to detect cholestasis of $98 \%$ and a specificity of $92 \%$ among 110 patients with hepatobiliary disease. ${ }^{101}$ Furthermore, the alkaline phosphatase liver isoenzyme had a positive predictive value of $90 \%$, a negative predictive value of $98 \%$, and a validity of $95 \%$ in this study. 


\section{Summary of the Differentiation between IFALD and NAFL/NASH}

Based on the evidence presented here, there are several characteristics that can be used to differentiate patients with IFALD and NAFL/NASH ( - Table 5 ):

1. High frequency of choline deficiency in IFALD versus choline sufficiency or excess in NAFL/NASH

2. High frequency of cholestasis in IFALD versus the absence of cholestasis in NAFL/NASH

3. High frequency of severe liver disease in IFALD versus low frequency in NAFL/NASH

4. Rapidly progressive liver disease with a poor prognosis for patients with IFALD versus those with NAFL/NASH

5. High frequency of malabsorption in patients with IFALD versus virtual nonexistence in those with NAFL/NASH

6. High frequency of microvesicular fat cells in IFALD versus an extremely low frequency in the fat cells of patients with NAFL/NASH

7. Obesity, metabolic syndrome, NIDDM, and hyperlipidemia are virtually nonexistent in patients with IFALD versus having a high frequency in those with NAFL/NASH.

8. Insulin resistance is a characteristic of patients with NAFLD, but insulin resistance is suppressed in conditions where there is choline deficiency and is not exacerbated by PN.

The medical literature substantiates the fact that IFALD is highly associated with PN and is a completely different disease than NAFL/NASH. Intestinal-failure-associated liver disease has a very different histology, pathogenesis, etiology, and epidemiology-and in addition, a substantially worse prognosis. In fact, AGA, ACG, and AASLD society guidelines specifically state that NAFL/NASH does not include IFALD. ${ }^{14}$

Cholestasis is a hallmark of most cases of IFALD, but is not present in NAFL/NASH. Microvesicular steatosis is common in IFALD and rare in NAFL/NASH. Metabolic risk factors (e.g., obesity, dyslipidemia, insulin resistance) that help define NAFL/NASH are not characteristic of patients who require long-term PN. The available data suggest that choline deficiency is a factor that leads to chronic IFALD and that choline deficiency is not a cause of, nor associated with, NAFL/NASH in humans. There is little potential for overlap between a diagnosis of IFALD and NAFL/NASH using the criteria described herein. The scientific evidence does not support the view that choline would be a useful therapy for NAFL/NASH, whereas there is a scientific basis for its use in IFALD. Therefore, it is concluded that IFALD is a distinct disease from NAFLD (i.e., NAFL and NASH).

\section{Main Concepts and Learning Points}

- Intestinal-failure-associated liver disease is characterized by the presence of cholestasis on liver biopsy OR an elevated serum alkaline phosphatase, AND/OR the presence of hepatic steatosis by histology OR imaging in the setting of intestinal failure with requirement of PN and in the absence of metabolic syndrome.

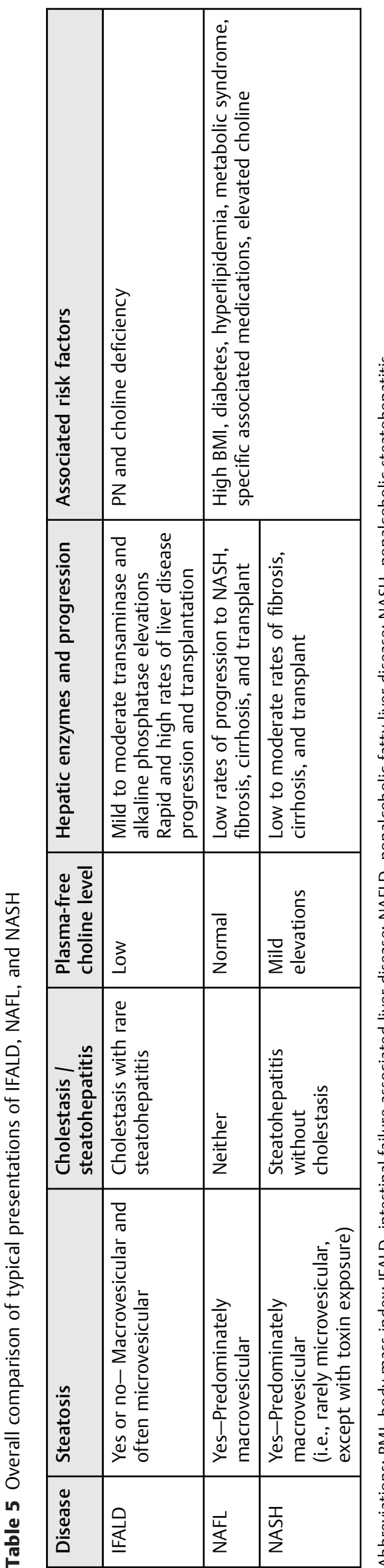


- Intestinal-failure-associated liver disease differs substantially from NAFLD, which is comprised of NAFL and NASH, on the basis of pathophysiology, risk factors, histology, epidemiology, prevalence, natural history, and prognosis.

- Plasma-free choline concentration is substantially decreased in IFALD and normal or elevated in NAFLD.

\section{Abbreviations}

$\begin{array}{ll}\text { AASLD } & \text { American Association for the Study of Liver Disease } \\ \text { AGA } & \text { American Gastroenterological Association } \\ \text { BMI } & \text { body mass index } \\ \text { ESLD } & \text { end-stage liver disease } \\ \text { HPN } & \text { home parenteral nutrition } \\ \text { IFALD } & \text { intestinal-failure-associated liver disease } \\ \text { IRTA } & \text { Intestinal Rehabilitation and Transplant } \\ & \text { Association } \\ \text { NAFLD } & \text { nonalcoholic fatty liver disease } \\ \text { NASH } & \text { nonalcoholic steatohepatitis } \\ \text { NIDDM } & \text { noninsulin-dependent diabetes mellitus } \\ \text { PN } & \text { parenteral nutrition }\end{array}$

\section{Author Contributions}

Drs. Buchman and Spilker conceived and drafted the manuscript; Dr. Naini developed the histological criteria and wrote portions of the histology section. All authors participated in the library investigation and critical revisions of the manuscript.

\section{Acknowledgments}

The authors wish to acknowledge the assistance of Naga Chalassani, MD, and Arun Sanyal, MD, for their insightful and critical review of the manuscript and helpful comments.

\section{References}

1 Caldwell SH, Al-Osaimi AMS, Argo CK. Nonalcoholic fatty liver disease. In: Schiff ER, Sorrell ME, Maddrey WC, eds. Schiff's Diseases of the Liver. $10^{\text {th }}$ ed. Philadelphia: Lippincott, Williams \& Wilkins; 2007:1117-1167

2 Lazo M, Hernaez R, Bonekamp S, et al. Non-alcoholic fatty liver disease and mortality among US adults: prospective cohort study. BMJ 2011;343:d6891

3 Buchman AL, Moukarzel A, Jenden DJ, Roch M, Rice K, Ament ME. Low plasma free choline is prevalent in patients receiving long term parenteral nutrition and is associated with hepatic aminotransferase abnormalities. Clin Nutr 1993;12(1):33-37

4 Compher CW, Kinosian BP, Stoner NE, Lentine DC, Buzby GP. Choline and vitamin B12 deficiencies are interrelated in folatereplete long-term total parenteral nutrition patients. JPEN J Parenter Enteral Nutr 2002;26(1):57-62

5 Pironi L, Arends J, Baxter J, et al; Home Artificial Nutrition \& Chronic Intestinal Failure; Acute Intestinal Failure Special Interest Groups of ESPEN. ESPEN endorsed recommendations. Definition and classification of intestinal failure in adults. Clin Nutr 2015; 34(2):171-180

6 Bowyer BA, Fleming CR, Ludwig J, Petz J, McGill DB. Does long-term home parenteral nutrition in adult patients cause chronic liver disease? JPEN J Parenter Enteral Nutr 1985;9(1): $11-17$

7 Stanko RT, Nathan G, Mendelow H, Adibi SA. Development of hepatic cholestasis and fibrosis in patients with massive loss of intestine supported by prolonged parenteral nutrition. Gastroenterology 1987;92(1):197-202

8 Chan S, McCowen KC, Bistrian BR, et al. Incidence, prognosis, and etiology of end-stage liver disease in patients receiving home total parenteral nutrition. Surgery 1999;126(1):28-34

9 Cavicchi M, Beau P, Crenn P, Degott C, Messing B. Prevalence of liver disease and contributing factors in patients receiving home parenteral nutrition for permanent intestinal failure. Ann Intern Med 2000;132(7):525-532

10 Beath S, Pironi L, Gabe S, et al. Collaborative strategies to reduce mortality and morbidity in patients with chronic intestinal failure including those who are referred for small bowel transplantation. Transplantation 2008;85(10):1378-1384

11 Buchman A. Total parenteral nutrition-associated liver disease. JPEN J Parenter Enteral Nutr 2002;26(5, Suppl)S43-S48

12 Buchman AL, Iyer K, Fryer J. Parenteral nutrition-associated liver disease and the role for isolated intestine and intestine/liver transplantation. Hepatology 2006;43(1):9-19

13 Kelly DA. Intestinal failure-associated liver disease: what do we know today? Gastroenterology 2006;130(2, Suppl 1)S70-S77

14 Chalasani N, Younossi Z, Lavine JE, et al. The diagnosis and management of non-alcoholic fatty liver disease: practice guideline by the American Gastroenterological Association, American Association for the Study of Liver Diseases, and American College of Gastroenterology. Gastroenterology 2012;142(7):1592-1609

15 Sanyal AJ, Brunt EM, Kleiner DE, et al. Endpoints and clinical trial design for nonalcoholic steatohepatitis. Hepatology 2011;54(1): 344-353

16 Kleiner DE, Brunt EM, Van Natta M, et al; Nonalcoholic Steatohepatitis Clinical Research Network. Design and validation of a histological scoring system for nonalcoholic fatty liver disease. Hepatology 2005;41(6):1313-1321

17 Naini BV, Lassman CR. Total parenteral nutrition therapy and liver injury: a histopathologic study with clinical correlation. Hum Pathol 2012;43(6):826-833

18 Lindor KDFC, Fleming CR, Abrams A, Hirschkorn MA. Liver function values in adults receiving total parenteral nutrition. JAMA 1979;241(22):2398-2400

19 Klein S, Nealon WH. Hepatobiliary abnormalities associated with total parenteral nutrition. Semin Liver Dis 1988;8(3):237-246

20 Quigley EM, Marsh MN, Shaffer JL, Markin RS. Hepatobiliary complications of total parenteral nutrition. Gastroenterology 1993;104(1):286-301

21 Mullick FGMC, Moran CA, Ishak KG. Total parenteral nutrition: a histopathologic analysis of the liver changes in 20 children. Mod Pathol 1994;7(2):190-194

22 Zambrano E, El-Hennawy M, Ehrenkranz RA, Zelterman D, ReyesMúgica M. Total parenteral nutrition induced liver pathology: an autopsy series of 24 newborn cases. Pediatr Dev Pathol 2004; 7(5):425-432

23 Kleiner DE. Liver injury due to total parenteral nutrition. In: Ferrell L, Kaker S, eds. Liver Pathology. New York: Demos Medical; 2011:235-236

24 Guglielmi FW, Regano N, Mazzuoli S, et al. Cholestasis induced by total parenteral nutrition. Clin Liver Dis 2008;12(1): 97-110, viii

25 Tandra S, Yeh MM, Brunt EM, et al; NASH Clinical Research Network (NASH CRN). Presence and significance of microvesicular steatosis in nonalcoholic fatty liver disease. J Hepatol 2011; 55(3):654-659

26 Chitturi S, Farrell GC. Drug-induced liver disease. In Schiff ER, Sorrell ME, Maddrey WC, eds. Schiff's Diseases of the Liver. $10^{\text {th }}$ ed. Philadelphia: Lippincott Williams and Wilkins; 2007:923-1003 
27 Hensley K, Kotake Y, Sang H, et al. Dietary choline restriction causes complex I dysfunction and increased $\mathrm{H}(2) \mathrm{O}(2)$ generation in liver mitochondria. Carcinogenesis 2000;21(5):983-989

28 Grattagliano I, Caraceni P, Calamita G, et al. Severe liver steatosis correlates with nitrosative and oxidative stress in rats. Eur J Clin Invest 2008;38(7):523-530

29 Teodoro JS, Rolo AP, Duarte FV, Simões AM, Palmeira CM. Differential alterations in mitochondrial function induced by a cholinedeficient diet: understanding fatty liver disease progression. Mitochondrion 2008;8(5-6):367-376

30 Schugar RC, Huang X, Moll AR, Brunt EM, Crawford PA. Role of choline deficiency in the Fatty liver phenotype of mice fed a low protein, very low carbohydrate ketogenic diet. PLoS One 2013; 8(8):e74806

31 Cotrim HP, De Freitas LA, Freitas C, et al. Clinical and histopathological features of NASH in workers exposed to chemicals with or without associated metabolic conditions. Liver Int 2004;24(2): 131-135

32 Caldwell SH, Patrie JT, Brunt EM, et al. The effects of 48 weeks of rosiglitazone on hepatocyte mitochondria in human nonalcoholic steatohepatitis. Hepatology2007b;46(4):1101-1107

33 Brunt EM. Nonalcoholic steatohepatitis. Semin Liver Dis 2004; 24(1):3-20

34 Brunt EM, Clouston AD. Histologic features of fatty liver disease. In: Bataller R, Caballeria J, eds. Nonalcoholic Steatohepatitis (NASH). Barcelona: Permanyer; 2007:95-110

35 Brunt EM. Pathology of nonalcoholic fatty liver disease. Nat Rev Gastroenterol Hepatol 2010;7(4):195-203

36 Yeh MM, Brunt EM. Pathology of nonalcoholic fatty liver disease. Am J Clin Pathol 2007;128(5):837-847

37 Burt AD, Mutton A, Day CP. Diagnosis and interpretation of steatosis and steatohepatitis. Semin Diagn Pathol 1998;15(4):246-258

38 Angulo P. Nonalcoholic fatty liver disease. N Engl J Med 2002; 346(16):1221-1231

39 Stegink LD, Den Besten L. Synthesis of cysteine from methionine in normal adult subjects: effect of route of alimentation. Science 1972;178(4060):514-516

40 Chawla RK, Berry CJ, Kutner MH, Rudman D. Plasma concentrations of transsulfuration pathway products during nasoenteral and intravenous hyperalimentation of malnourished patients. Am J Clin Nutr 1985;42(4):577-584

41 Sentongo TA, Kumar P, Karza K, Keys L, Iyer K, Buchman AL. Whole-blood-free choline and choline metabolites in infants who require chronic parenteral nutrition therapy. J Pediatr Gastroenterol Nutr 2010;50(2):194-199

42 Riedijk MA, Stoll B, Chacko S, et al. Methionine transmethylation and transsulfuration in the piglet gastrointestinal tract. Proc Natl Acad Sci U S A 2007;104(9):3408-3413

43 Waterhouse C, Clarke EF, Heinig RE, Lewis AM, Jeanpretre N. Free amino acid levels in the blood of patients undergoing parenteral alimentation. Am J Clin Nutr 1979;32(12):2423-2429

44 Dahlström KA, Ament ME, Laidlaw SA, Kopple JD. Plasma amino acid concentrations in children receiving long-term parenteral nutrition. J Pediatr Gastroenterol Nutr 1988;7(5):748-754

45 Tayek JA, Bistrian B, Sheard NF, Zeisel SH, Blackburn GL. Abnormal liver function in malnourished patients receiving total parenteral nutrition: a prospective randomized study. J Am Coll Nutr 1990; 9(1):76-83

46 Buchman AL, Dubin M, Jenden D, et al. Lecithin increases plasma free choline and decreases hepatic steatosis in long-term total parenteral nutrition patients. Gastroenterology 1992;102(4 Pt 1):1363-1370

47 Buchman AL, Dubin MD, Moukarzel AA, et al. Choline deficiency: a cause of hepatic steatosis during parenteral nutrition that can be reversed with intravenous choline supplementation. Hepatology 1995;22(5):1399-1403

48 Buchman AL, Ament ME, Sohel M, et al. Choline deficiency causes reversible hepatic abnormalities in patients receiving parenteral nutrition: proof of a human choline requirement: a placebocontrolled trial. JPEN J Parenter Enteral Nutr2001b;25(5): 260-268

49 Spencer MD, Hamp TJ, Reid RW, Fischer LM, Zeisel SH, Fodor AA. Association between composition of the human gastrointestinal microbiome and development of fatty liver with choline deficiency. Gastroenterology 2011;140(3):976-986

50 Rinella ME, Elias MS, Smolak RR, Fu T, Borensztajn J, Green RM. Mechanisms of hepatic steatosis in mice fed a lipogenic methionine choline-deficient diet. J Lipid Res 2008;49(5): 1068-1076

$51 \mathrm{Mu}$ YP, Ogawa T, Kawada N. Reversibility of fibrosis, inflammation, and endoplasmic reticulum stress in the liver of rats fed a methionine-choline-deficient diet. Lab Invest 2010;90(2): 245-256

52 Kaminski DL, Adams A, Jellinek M. The effect of hyperalimentation on hepatic lipid content and lipogenic enzyme activity in rats and man. Surgery 1980;88(1):93-100

53 Raubenheimer PJ, Nyirenda MJ, Walker BR. A choline-deficient diet exacerbates fatty liver but attenuates insulin resistance and glucose intolerance in mice fed a high-fat diet. Diabetes 2006; 55(7):2015-2020

54 Macfarlane DP, Zou X, Andrew R, et al. Metabolic pathways promoting intrahepatic fatty acid accumulation in methionine and choline deficiency: implications for the pathogenesis of steatohepatitis. Am J Physiol Endocrinol Metab 2011;300(2): E402-E409

55 Veteläinen R, van Vliet A, van Gulik TM. Essential pathogenic and metabolic differences in steatosis induced by choline or methione-choline deficient diets in a rat model. J Gastroenterol Hepatol 2007;22(9):1526-1533

56 Niebergall LJ, Jacobs RL, Chaba T, Vance DE. Phosphatidylcholine protects against steatosis in mice but not non-alcoholic steatohepatitis. Biochim Biophys Acta 2011;1811(12):1177-1185

57 Imajo K, Fujita K, Yoneda M, et al. Plasma free choline is a novel non-invasive biomarker for early-stage non-alcoholic steatohepatitis: A multi-center validation study. Hepatol Res 2012;42(8): 757-766

58 Imajo K, Yoneda M, Fujita K, et al. Oral choline tolerance test as a novel noninvasive method for predicting nonalcoholic steatohepatitis. J Gastroenterol 2014;49(2):295-304

59 Nehra V, Angulo P, Buchman AL, Lindor KD. Nutritional and metabolic considerations in the etiology of nonalcoholic steatohepatitis. Dig Dis Sci 2001;46(11):2347-2352

60 Serviddio G, Giudetti AM, Bellanti F, et al. Oxidation of hepatic carnitine palmitoyl transferase-I (CPT-I) impairs fatty acid betaoxidation in rats fed a methionine-choline deficient diet. PLoS One 2011;6(9):e24084

61 Cheung O, Sanyal AJ. Recent advances in nonalcoholic fatty liver disease. Curr Opin Gastroenterol 2010;26(3):202-208

62 Andersen T, Gluud C. Liver morphology in morbid obesity: a literature study. Int J Obes1984a;8(2):97-106

63 Andersen T, Christoffersen P, Gluud C. The liver in consecutive patients with morbid obesity: a clinical, morphological, and biochemical study. Int J Obes1984b;8(2):107-115

64 Dixon JB, Bhathal PS, O'Brien PE. Nonalcoholic fatty liver disease: predictors of nonalcoholic steatohepatitis and liver fibrosis in the severely obese. Gastroenterology 2001;121(1):91-100

65 García-Monzón C, Martín-Pérez E, Iacono OL, et al. Characterization of pathogenic and prognostic factors of nonalcoholic steatohepatitis associated with obesity. J Hepatol 2000;33(5): 716-724

66 Tjellesen L, Staun M, Nielsen PK. Body composition changes measured by dual-energy X-ray absorptiometry in patients receiving home parenteral nutrition. Scand J Gastroenterol 1997;32(7):686-690

67 Wei JL, Leung JCF, Loong TCW, et al. Prevalence and severity of nonalcoholic fatty liver disease in non-obese patients: a 
population study using proton-magnetic resonance spectroscopy. Am J Gastroenterol 2015;110(9):1306-1314, quiz 1315

68 Marchesini G, Brizi M, Morselli-Labate AM, et al. Association of nonalcoholic fatty liver disease with insulin resistance. Am J Med 1999;107(5):450-455

69 Sanyal AJ, Campbell-Sargent C, Mirshahi F, et al. Nonalcoholic steatohepatitis: association of insulin resistance and mitochondrial abnormalities. Gastroenterology 2001;120(5):1183-1192

70 Falchuk KR, Fiske SC, Haggitt RC, Federman M, Trey C. Pericentral hepatic fibrosis and intracellular hyalin in diabetes mellitus. Gastroenterology 1980;78(3):535-541

71 Silverman JF, Pories WJ, Caro JF. Liver pathology in diabetes mellitus and morbid obesity. Clinical, pathological, and biochemical considerations. Pathol Annu 1989;24(Pt 1):275-302

72 Zareba K, Czygier M, Kamocki Z, Cepowicz D, Szmitkowski M, Kedra B. Parenteral nutrition and preOp preparation in prevention of post-operative insulin resistance in gastrointestinal carcinoma. Adv Med Sci 2013;58(1):150-155

73 Mukherjee K, Sowards KJ, Brooks SE, et al. Insulin resistance in critically injured adults: contribution of pneumonia, diabetes, nutrition, and acuity. Surg Infect (Larchmt) 2015;16(5):490-497

74 Svanfeldt M, Thorell A, Nygren J, Ljungqvist O. Postoperative parenteral nutrition while proactively minimizing insulin resistance. Nutrition 2006;22(5):457-464

75 Lienhardt A, Rakotoambinina B, Colomb V, et al. Insulin secretion and sensitivity in children on cyclic total parenteral nutrition. JPEN J Parenter Enteral Nutr 1998;22(6):382-386

76 Assy N, Kaita K, Mymin D, Levy C, Rosser B, Minuk G. Fatty infiltration of liver in hyperlipidemic patients. Dig Dis Sci 2000; 45(10):1929-1934

77 Chitturi S, Abeygunasekera S, Farrell GC, et al. NASH and insulin resistance: insulin hypersecretion and specific association with the insulin resistance syndrome. Hepatology 2002;35(2):373-379

78 Park YW, Zhu S, Palaniappan L, Heshka S, Carnethon MR, Heymsfield SB. The metabolic syndrome: prevalence and associated risk factor findings in the US population from the Third National Health and Nutrition Examination Survey, 1988-1994. Arch Intern Med 2003;163(4):427-436

79 Liangpunsakul S, Chalasani N. Unexplained elevations in alanine aminotransferase in individuals with the metabolic syndrome: results from the third National Health and Nutrition Survey (NHANES III). Am J Med Sci 2005;329(3):111-116

80 Pagano G, Pacini G, Musso G, et al. Nonalcoholic steatohepatitis, insulin resistance, and metabolic syndrome: further evidence for an etiologic association. Hepatology 2002;35(2):367-372

81 Abdal Raheem S, Deen OJ, Corrigan ML, et al. Bariatric surgery complications leading to small bowel transplant: a report of 4 cases. JPEN J Parenter Enteral Nutr 2014;38(4):513-517

82 Abbott WM, Abel RM, Fischer JE. The effects ot total parenteral nutrition upon serum lipid levels. Surg Gynecol Obstet 1976; 142(4):565-568

83 Badimon JJ, Fleming CR, Patton J, Mao SJ. Changes of plasma levels of apolipoproteins A-I, A-II, and B and their isoforms in patients with intestinal failure receiving long-term parenteral nutrition. Am J Clin Nutr 1987;45(2):414-422

84 Howard L, Ament M, Fleming CR, Shike M, Steiger E. Current use and clinical outcome of home parenteral and enteral nutrition therapies in the United States. Gastroenterology 1995;109(2):355-365

85 Howard L, Heaphey L, Fleming CR, Lininger L, Steiger E. Four years of North American registry home parenteral nutrition outcome data and their implications for patient management. JPEN J Parenter Enteral Nutr 1991;15(4):384-393

86 Pironi L, Goulet O, Buchman A, et al; Home Artificial Nutrition and Chronic Intestinal Failure Working Group of ESPEN. Outcome on home parenteral nutrition for benign intestinal failure: a review of the literature and benchmarking with the European prospective survey of ESPEN. Clin Nutr 2012;31(6): 831-845

87 Rivera A Jr, Bhatia J, Rassin DK, Gourley WK, Catarau E. In vivo biliary function in the adult rat: the effect of parenteral glucose and amino acids. JPEN J Parenter Enteral Nutr 1989;13(3): 240-245

88 Duerksen DR, Van Aerde JE, Chan G, Thomson AB, Jewell LJ, Clandinin MT. Total parenteral nutrition impairs bile flow and alters bile composition in newborn piglet. Dig Dis Sci 1996;41(9): 1864-1870

89 Vanwijngaerden YM, Langouche L, Derde S, et al. Impact of parenteral nutrition versus fasting on hepatic bile acid production and transport in a rabbit model of prolonged critical illness. Shock 2014;41(1):48-54

90 Messing B, Bories C, Kunstlinger F, Bernier JJ. Does total parenteral nutrition induce gallbladder sludge formation and lithiasis? Gastroenterology 1983;84(5 Pt 1):1012-1019

91 Balistreri WF, Bove KE. Hepatobiliary consequences of parenteral alimentation. Prog Liver Dis 1990;9:567-601

92 LeBlanc MJ, Gavino V, Pérea A, Yousef IM, Lévy E, Tuchweber B. The role of dietary choline in the beneficial effects of lecithin on the secretion of biliary lipids in rats. Biochim Biophys Acta 1998; 1393(2-3):223-234

93 Buchman AL. Short bowel syndrome. In: Feldman M, Friedman LS, Brandt LJ, eds. Sleisenger and Fortran's Gastrointestinal Disease. 9th ed. Philadelphia: Saunders ; 2010:1779-1795

94 Fiel MI, Wu HS, Iyer K, Rodriguez-Laiz G, Schiano TD. Rapid reversal of parenteral-nutrition-associated cirrhosis following isolated intestinal transplantation. J Gastrointest Surg 2009; 13(9):1717-1723

95 Fielding CM, Angulo P. Hepatic steatosis and steatohepatitis: are they really two distinct entities? Curr Hepatol Rep 2014;13(2): 151-158

96 Adams LA, Lymp JF, St Sauver J, et al. The natural history of nonalcoholic fatty liver disease: a population-based cohort study. Gastroenterology 2005;129(1):113-121

97 Dam-Larsen S, Franzmann M, Andersen IB, et al. Long term prognosis of fatty liver: risk of chronic liver disease and death. Gut 2004;53(5):750-755

98 Haflidadottir S, Jonasson JG, Norland H, et al. Long-term followup and liver-related death rate in patients with non-alcoholic and alcoholic related fatty liver disease. BMC Gastroenterol 2014; 14:166(Abst.)

99 McPherson S, Hardy T, Henderson E, Burt AD, Day CP, Anstee QM. Evidence of NAFLD progression from steatosis to fibrosing-steatohepatitis using paired biopsies: implications for prognosis and clinical management. J Hepatol 2015;62(5): 1148-1155

100 Reichling JJ, Kaplan MM. Clinical use of serum enzymes in liver disease. Dig Dis Sci 1988;33(12):1601-1614

101 Ricci OE, Tozzi P, Caldini M, et al. Serum alkaline phosphatase isoenzymes: the fast liver fraction in the diagnosis of hepatobiliary disease with or without cholestasis. Boll Soc Ital Biol Sper 1984;60(7):1363-1368 